\title{
OPTICAL PROPERTIES OF AN ELECTRON BOUND TO A DOUBLE WELL POTENTIAL IN A DIELECTRIC*
}

\author{
M. A. AEGERTER** and F. L ÜTY \\ Physics Deparment, University of Utah, Salt Lake Citl, Utah 84/12, USA
}

\begin{abstract}
The absorption and emission properties of $\mathrm{F}$ ' centers in $\mathrm{KCl}$ (two adjacent anion vacancies which bind onc electron) were investigated between 0.6 and $5 \mathrm{eV}$ using an optically aligned center system. Energy positions, level degeneracies, polarization behavior and oscillator strengths of 8 absorption transitions were found in close agreement to the calculated states of the $\mathrm{H}_{2}$ : molecular ion corrected for a single dielectric constant and the proper separation between the wo effective positive charges. Excitation of the lowest $2 p^{\prime \prime}$ state at $1.38 \mu \mathrm{m}$ leads with temperature independent quantum efficiency to a Stokes shifted emission at $1.67 \mathrm{\mu m}$ almost in mirror symmetry with the zero phonon line at $1.5260 \mathrm{\mu m}$. Excitation of all higher absorption transitions ( $2 p_{.} \tau_{1}$, etc.) at liquid helium temperature (LHeT) produces a visible luminescence at $600 \mathrm{~mm}$. At higher temperature a thermally activated process (activation energy IE $0.063 \mathrm{eV}$ ) transfers the excitation energy to the lowest excited state $2 p_{n}$ quenching the $600 \mathrm{~nm}$ luminescence and producing the IR emission.
\end{abstract}

\section{Introduction}

In an alkali halide crystal, the " $\mathrm{F}_{2}^{+}$center" consists of an electron bound to the double well potential of two adjacent anion vacancies in a $\langle 110\rangle$ direction. Its model was often proposed in the past few years in order to explain the origin of several optical transitions observed in these materials. The first unambiguous identification of the lowest optical absorption of the $\mathrm{F}_{2}^{+}$center was done in $\mathrm{KCl}, \mathrm{KBr}$ and $\mathrm{Kl}$ by Schneider and Rabin ${ }^{\prime}$ ) in 1965. At present the location of these transitions, which generally exhibit a small zero phonon line is also known in $\mathrm{NaF}, \mathrm{LiF}$ and $\mathrm{NaCl}^{2}$ ). Aegerter and Lüty ${ }^{3}$ ) studied in detail the formation and bleaching kinetics of this center in $\mathrm{KCl}$ crystals. They showed that at low temperature, the $\mathrm{F}_{2}^{+}$center could be created by optical ionization of $M\left(=F_{2}\right)$ centers (two electrons shared by two adjacent anion vacancies) in the UV region. In an additively colored crystal it is found that the electrons removed from the $M$ centers are trapped either in an $F$ or $M$ center, creating therefore $F^{\prime}$ or $M^{\prime}$ centers. In this case

* Work supported by the U.S. Air Force Office of Scientific Rescarch under Grant No. AFOSR-69-1645.

*: Partly sponsored by a postdoctoral grant of F.N.S.R.S. (Switzerland).

Present address: Institut de Physique, Université de Neuchâtel, Neuchảtel (Switzerland). 
these excess electron centers stabilize the $\mathrm{F}_{2}^{+}$centers. The concentration of the $\mathrm{F}_{2}^{+}$centers created by this process is very low $\left(<8 \times 10^{15} \mathrm{~cm}^{-3}\right)$. However the introduction of extra electron traps into the crystal can increase the amount of $\mathrm{F}_{2}^{+}$centers and concentrations up to $2 \times 10^{17} \mathrm{~cm}^{-3}$ have been achieved. This is done for instance by X-raying the crystal at LHeT in order to create intrinsic Frenkel pairs (interstitial anion-anion vacancy pairs) or by doping the $\mathrm{KCl}$ crystal with $\mathrm{KH}$ in order to create by photochemical reaction at low temperature pairs of interstitial hydrogen ion-anion vacancy.

\section{Near infrared transitions}

The fig. 1 shows the near IR absorption spectra of the $\mathrm{F}_{2}^{+}$centers in $\mathrm{KCl}$ at $\mathrm{LHeT}, \mathrm{LNT}$ and $150^{\circ} \mathrm{K}$. At LHeT the shape of the band is strongly asymmetric. Its maximum occurs at $1.4 \mu \mathrm{m}$ and its width measured at half maximum is $0.0825 \mathrm{eV}$. A zero phonon line, not fully resolved in our measurement, appears at $1.5262 \mu \mathrm{m}$ in good agreement with Pierce $\left.{ }^{4}\right)$. The observed dichroism is identical to the first transition of the $M$ centers at $800 \mathrm{~nm}$ and

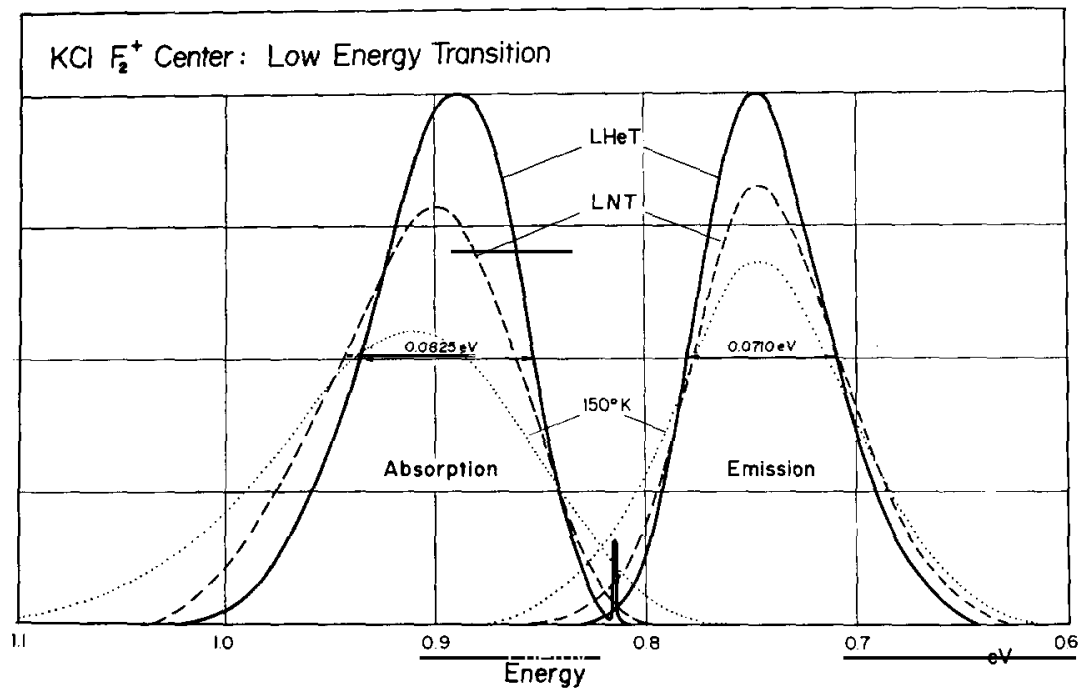

Fig. 1. The near infrared optical transitions of the $\mathrm{F}_{2}+$ center in $\mathrm{KCl}$ at three different temperatures: LHeT, LNT and $150^{\circ} \mathrm{K}$. The absorption spectra (left side) are as recorded with the height of the maximum of the band measured at LHeT normalized to 1 . The zero phonon line observed in absorption at $15262 \AA$ at LHeT is not fully resolved.

The right side of the picture shows the corrected emission spectra as observed if an equal number of $\mathrm{F}_{2}+$ centers are excited near $1.4 \mu \mathrm{m}$. The zeroth moment of the emission bands is therefore a measurement of the relative quantum emission efficiency. 
shows consequently that the center has a $\langle 110\rangle$ axis of symmetry. Its optical dipole moment indicates furthermore that the transition is fully $\sigma$ polarized.

Optical excitation in this band produces a luminescence. the maximum of which occurs at $1.67 \mu \mathrm{m}$ at LHeT (fig. 1). The band has almost mirror symmetry to the absorption band respective to the zero phonon line. its width at half maximum being $0.0716 \mathrm{eV}$. This near IR emission is also fully $\sigma$ polarized $(\langle 110\rangle$ direction $)$ which is consistent with the model of the center.

\section{Visible and near UV transitions}

When excited in the visible and near $U V$ region at $L$ HeT the $\mathrm{F}_{2}^{+}$center in $\mathrm{KCl}$ produces a red luminescence centered at $600 \mathrm{~nm}$ which has a width at half maximum of $0.128 \mathrm{eV}$. Fig. 2 shows a plot of the relative intensity of this unique emission under three different excitations (505, 492 and $390 \mathrm{~nm}$ ).

The dichroic properties of the excitation spectrum of this luminescence were studied at LHeT with an almost perfectly aligned $\mathrm{F}_{2}^{+}$center system. The transition centered around $500 \mathrm{~nm}$ was found as the superposition of two strongly overlapping bands with their maximum occurring respectively at $493 \mathrm{~nm}$ (half width $0.138 \mathrm{eV}$ ) and $509 \mathrm{~nm}$ (half width $0.112 \mathrm{eV}$ ). Their

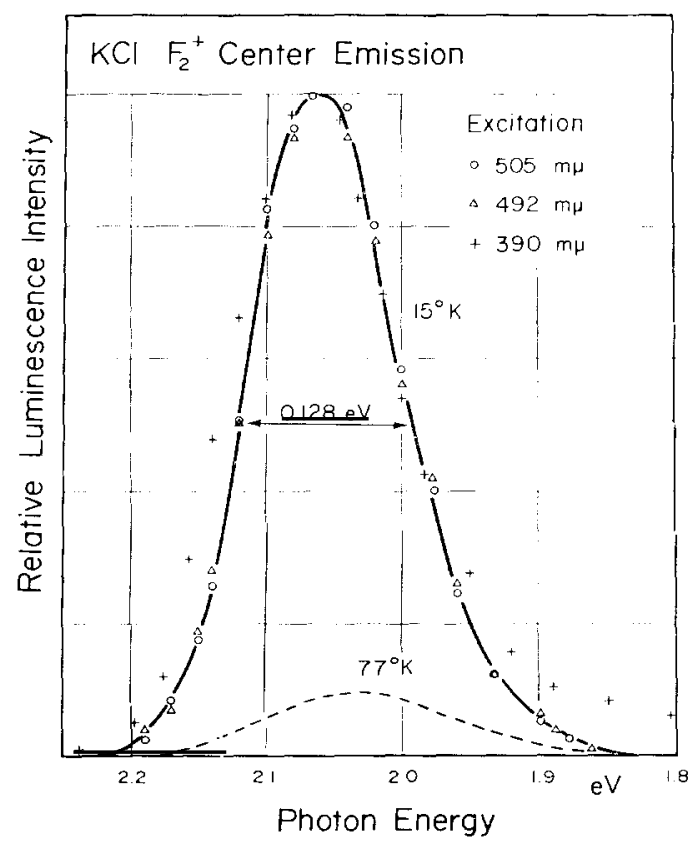

Fig. 2. Relative intensity of the visible $F_{2}$ emission in $\mathrm{KCl}$ for excitation at: $505 \mathrm{~nm}$. $492 \mathrm{~nm}, \quad 390 \mathrm{~nm}$ respectively at 15 and $77 \mathrm{~K}$. 
relative height after adequate correction and normalization is found in a $3: 4$ ratio. Furthermore the $493 \mathrm{~nm}$ excitation band has an optical dipole moment in a $\langle 110\rangle$ direction, perpendicular to the symmetry axis of the center. On the other hand, the component at $509 \mathrm{~nm}$ has its optical dipole moment along a $\langle 100\rangle$ direction perpendicular to the center axis. These two transitions thus belong to a $\pi$-like state, its twofold degeneracy being lifted by the crystalline field.

Identical measurements were repeated for the two order of magnitude weaker near UV excitation bands (in the $390 \mathrm{~nm}$ region). At least five welldefined transitions have been resolved out of this complex structure. The

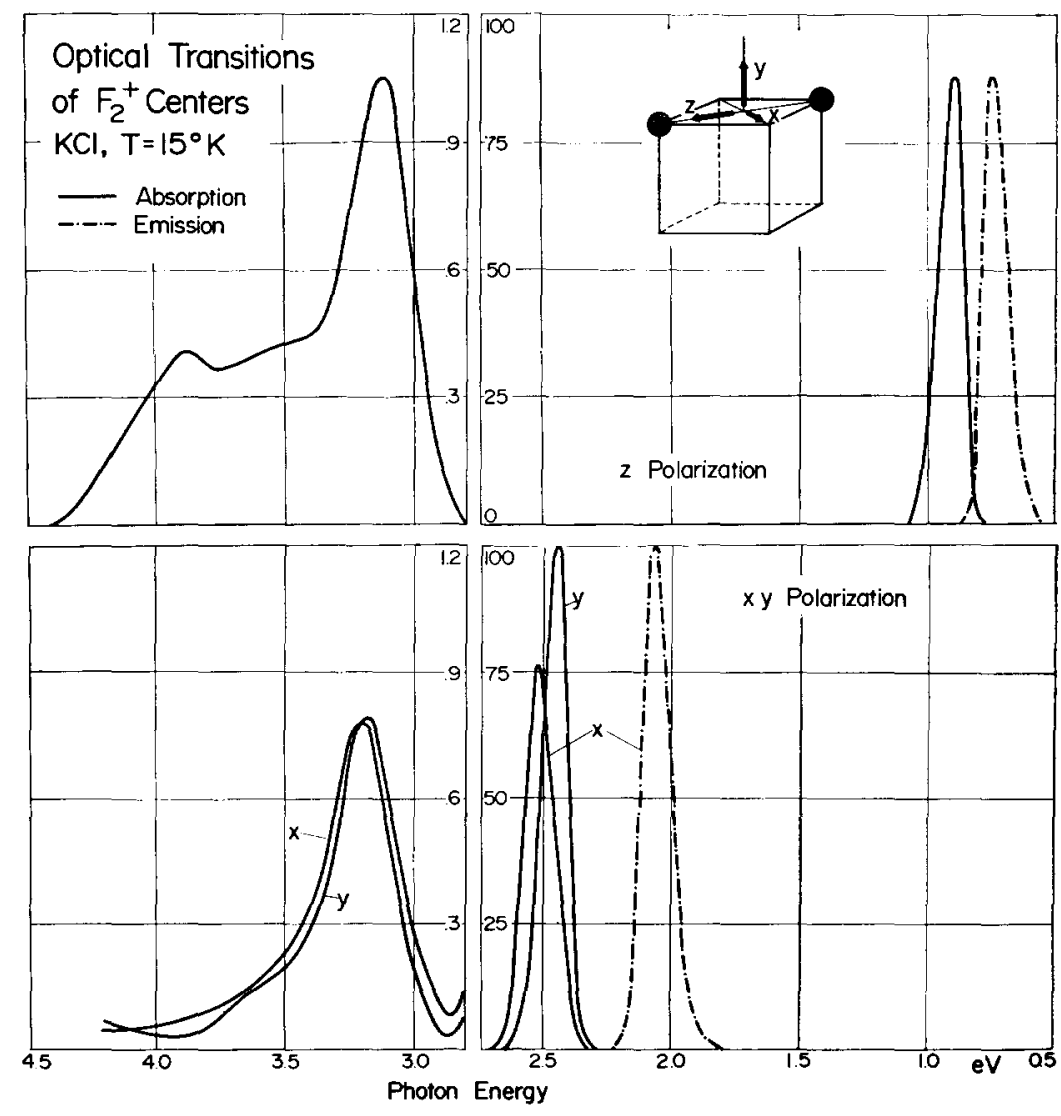

Fig. 3. The optical transitions of $\mathrm{F}_{2}{ }^{+}$centers in $\mathrm{KCl}$ measured at $15^{\circ} \mathrm{K}$. The upper part of the figure summarizes the $\sigma$-type polarized transitions, while the lower part represents the $\pi$-type transitions. The height of each excitation (or absorption) band is normalized to the same number of centers excited with light polarized parallel to their optical dipole moment. Note the change of scale between the near UV and the visible and near IR transitions. 
$390 \mathrm{~nm}$ band was found to be the superposition of one $\sigma$ and two $\pi$-like transitions respectively peaking at 396 and $388 \mathrm{~nm}$ with their width at half maximum approximately $0.3 \mathrm{eV}$ for the three of them. At higher energy the observed spectrum is still weaker and mostly defined by two $\sigma$ polarized transitions around 342 and $315 \mathrm{~nm}$.

Whatever is the orientation of the optical dipole moment of the visible or near UV excitation, the $600 \mathrm{~nm}$ luminescence is always found polarized in the $\langle 110\rangle$ direction perpendicular to the symmetry axis of the center. Fig. 3 gives a complete summary of the positions, relative height, half width and polarization of all the optical transitions so far measured for the $\mathrm{F}_{2}^{+}$ center in $\mathrm{KCl}$ near LHeT.

\section{The $\mathrm{F}_{2}^{+}$transitions at higher temperature}

As shown on fig. I, the near IR absorption and emission bands broaden and shift when the temperature is increased. Their width at half maximum $H(T)$ can be described with a satisfactory agreement by:

$$
H^{2}(T)=H^{2}(0) \operatorname{coth}\left(\begin{array}{c}
h \omega \\
2 k T
\end{array}\right) \text {. }
$$

with an effective frequency respectively given by:

$$
\begin{aligned}
& ()_{\mathrm{a}}=2 \pi \times 2.3 \times 10^{12} \mathrm{sec}^{-1}(0.0095 \mathrm{eV}) \\
& \omega_{\mathrm{e}}=2 \pi \times 3.4 \times 10^{12} \mathrm{sec}^{-1}(0.014 \mathrm{eV})
\end{aligned}
$$

Contrary to other known color center transitions in the alkali halide, the near IR absorption band of the $\mathrm{F}_{2}^{+}$center shifts to higher energy with rising temperature.

Furthermore a careful analysis shows, within the experimental errors, that for a given constant number of centers the value of the integrated emission measured up to $180^{\circ} \mathrm{K}$ remains the same. This shows that the quantum efficiency of the near IR emission is constant and may have a value of one over the whole temperature range, because radiationless processes. if present, would likely have an increasing probability with temperature.

The $600 \mathrm{~nm}$ visible luminescence which is excited by all visible and near UV transitions shows a completely different behavior. Its integrated intensity decreases rapidly with the temperature to become unmeasurable over $100 \mathrm{~K}$. However during the quenching of the visible luminescence we observe in a complementary way the appearance of the near IR emission under the same visible and UV excitation (fig. 4). 


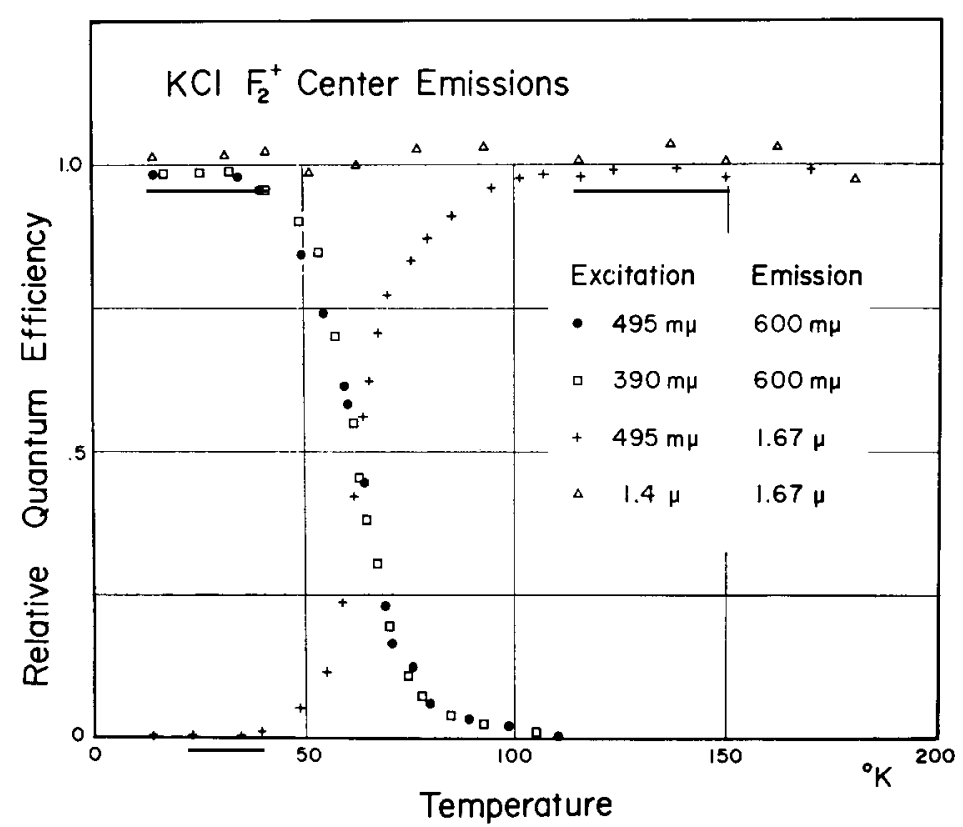

Fig. 4. Relative quantum efficiencies of the $\mathrm{F}_{2}{ }^{+}$center emissions in $\mathrm{KCl}$ in function of temperature

$\begin{array}{cc}\text { Excitation } & \text { Emission } \\ 495 \mathrm{~nm} & 600 \mathrm{~nm} \\ 390 \mathrm{~nm} & 600 \mathrm{~nm} \\ 495 \mathrm{~nm} & 1.67 \mu \mathrm{m} \\ 1.4 \mu \mathrm{m} & 1.67 \mu \mathrm{m}\end{array}$

This behavior can be fitted by a simple expression which describes the thermal quenching of the red luminescence by

$$
\eta_{\mathrm{red}}=\frac{1}{1+v \tau \exp (-\Delta E / k T)}
$$

and the simultaneous appearance of the near IR luminescence by

$$
\eta_{\mathrm{IR}}=\frac{1}{1+(v \tau)^{-1} \exp \overline{(\Delta E / k T)} .}
$$

A best fit to both expressions is obtained for $\Delta E=0.063 \mathrm{eV}$ and $v \tau=$ $1.2 \times 10^{5}$. All the described luminescence properties of the $\mathrm{F}_{2}^{+}$center can be summarized in the following way (see fig. 5 , right side): Optical excitation into the lowest excited state $(1.4 \mu \mathrm{m})$ leads independent of temperature with a constant (possibly full) quantum efficiency to the near IR $1.67 \mu \mathrm{m}$ emission polarized in the axis of the center. After optical excitation into all the higher 


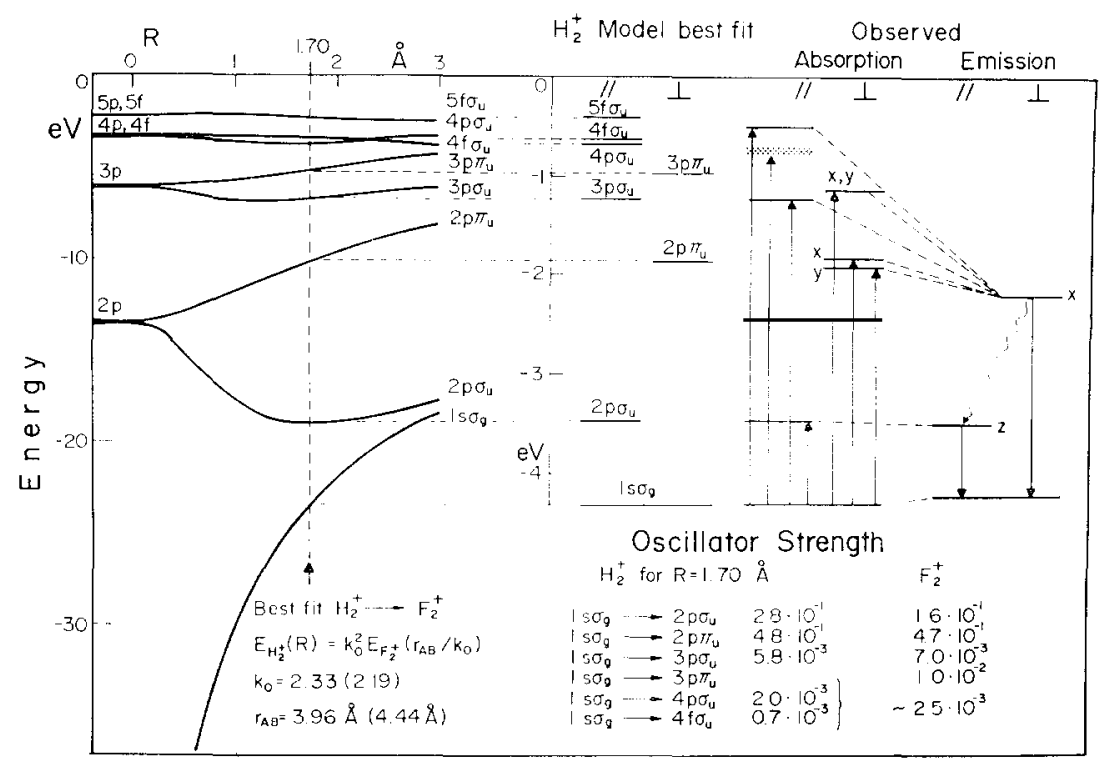

Fig. 5. Analogy between the $\mathrm{F}_{2}$ center in $\mathrm{KCl}$ and the $\mathrm{H}_{2}$ molecule. The lelt side shows the energy scheme of the ground state $\mathrm{Is}_{\mathrm{s}}$ and the higher u-type levels of the $\mathrm{H}_{2}$ molecule in function of the distance of the two protons. The energy levels of the $\mathrm{F}_{2}{ }^{\prime}$ eenter in $\mathrm{KCl}$ are related to the energy levels of the $\mathrm{H}_{2}$ molecule by $E_{\mathrm{H}}(R) \quad k_{11}{ }^{2} E_{1}:\left(r_{11} / k(1)\right)$. The best fit, shown on the right side of the figure, is obtained with $R \quad 1.70 \AA$, which determines the value of the dielectric constant of the crystal $k_{11} 2.33$ and the distance between the two anionic vacancies of the $F_{2}$ center $r_{1 / 3} \quad 3.96 \AA$ (usual values $k_{0}$ ) 2.19 and $r_{113} \quad 4.44 \AA$ respectively). The values of the oscillator strengths of the observed $\mathrm{F}_{2}$ ' transitions are also in good agreement with the calculated values for the $\mathrm{H}_{2}$ molecule for $R \quad 1.70 \AA$.

excited states, the excitation energy is always transferred radiationless into the lowest relaxed excited state which is polarized perpendicular to the center axis. From here it is emitted at low temperature as the $600 \mathrm{~nm}$ luminescence polarized in the $\langle 110\rangle$ direction perpendicular to the center axis. A temperature activated radiationless process (activation energy $\Delta E=$ $0.063 \mathrm{eV}$ ) competes with this radiative process, quenching the red luminescence around LNT. This radiationlessprocess obviously transfers the excitition energy into the lowest excited state, where it now appears as the $\sigma$ polarized IR emission. An estimation of the radiative lifetime $\tau$ for both emissions can be given by using a theoretical relation derived from the Einstein relation by Fowler and Dexter ${ }^{5}$ ) and valid for any luminescent center in ionic crystals. Since in both cases the emission energies are close to the absorption energies (small Stokes shift) the electronic matrix elements for absorption and emission may be assumed to be equal. In the limit of this approximation an expected lifetime for the $600 \mathrm{~nm}$ emission of $20 \mathrm{~ns}$ and 
$150 \mathrm{~ns}$ for the near IR emission is obtained. With this lifetime value for the red luminescence the attempt frequency for the competing radiationless process is obtained (from the measured $v \tau=1.2 \times 10^{5}$ ) to be $v=6 \times 10^{12}$ $\sec ^{-1}$.

\section{Analogy with the $\mathrm{H}_{2}^{+}$molecular ion}

Herman, Wallis and Wallis ${ }^{6}$ ) derived a simple theoretical model by considering the $\mathrm{F}_{2}^{+}$center in alkali halide crystal as an $\mathrm{H}_{2}^{+}$molecular ion embedded in an dielectric medium characterized by a dielectric constant $k_{0}$. The energy $E\left(r_{\mathrm{AB}}, k_{0}\right)$ of a given electronic state of the hydrogen molecular ion immersed in a dielectric medium (or the $\mathrm{F}_{2}^{+}$center in the crystal) may thus be connected to the energy $E_{\mathrm{H}_{2}+}(R)$ of the same electronic state for the same molecule in vacuum by:

$$
E_{\mathrm{F}_{2}+}\left(r_{\mathrm{AB}}, k_{0}\right)=\frac{1}{k_{0}^{2}} E_{\mathrm{H}_{2}+}(R)
$$

with $R=r_{\mathrm{AB}} / k_{0}, r_{\mathrm{AB}}=$ distance between the two anionic vacancies.

An extensive tabulation over a wide range of internuclear distances of ten calculated energy levels of the hydrogen molecular ion has been given by Bates et al. ${ }^{7}$ ) and Gilbert ${ }^{8}$ ).

The left side of fig. 5 shows a plot in function of the internuclear distance of the $1 \mathrm{~s} \sigma_{\mathrm{g}}$ ground state and the calculated higher level with ungerade parity $\left(2 \mathrm{p} \sigma_{\mathrm{u}}, 2 \mathrm{p} \pi_{\mathrm{u}}, 3 \mathrm{p} \sigma_{\mathrm{u}}, 3 \mathrm{p} \pi_{\mathrm{u}}, 4 \mathrm{p} \sigma_{\mathrm{u}}, 4 \mathrm{f} \sigma_{\mathrm{u}} \ldots\right)$ which will give rise to an allowed electronic transition.

The relative energy separations of the observed absorption transitions of the $\mathrm{F}_{2}^{+}$center were best fitted in this diagram. With the internuclear distance thus determined ( $R=1.70 \AA)$, we can determine with the relation (4) an effective dielectric constant $k_{0}=2.33$ and the distance between the two anionic vacancies $r_{\mathrm{AB}}=3.96 \AA$. These calculated values are in close agreement with the values accepted for $\mathrm{KCl}$, respectively 2.19 and $4.44 \AA$.

On the right side of the figure, the observed absorption transitions of the $\mathrm{F}_{2}^{+}$center are shown together with the $\mathrm{H}_{2}^{+}$model energy levels best fit. The agreement for the location and the polarization of the $\sigma$ type bands is remarkable over the whole energy range. The $1.4 \mu \mathrm{m}$ and the $396 \mathrm{~nm}$ transitions may be unambiguously described as transitions from the $1 \mathrm{~s} \sigma_{\mathrm{g}}$ state to respectively the $2 \mathrm{p} \sigma_{\mathrm{u}}$ and $3 \mathrm{p} \sigma_{\mathrm{u}}$ states. At higher energy the small $342 \mathrm{~nm}$ band appears likely to be the superposition of transitions to the $4 \mathrm{p} \sigma_{\mathrm{u}}$ and $4 \mathrm{f} \sigma_{\mathrm{u}}$ states while the last one peaking around $315 \mathrm{~nm}$ is probably the envelope of all the $\sigma$-like allowed transitions having a quantum number $n \geq 5$ and converging to the ionization limit at $4.4 \mathrm{eV}(282 \mathrm{~nm})$. 
Using the same best fit the agreement for the energy positions of the $\pi$-like transitions is also found relatively good, the 493 and $509 \mathrm{~nm}$ bands correspond without any doubt to the $1 \mathrm{~s} \sigma_{\mathrm{g}} \rightarrow 2 \mathrm{p} \pi_{\mathrm{u}}$ transition, which is split by the crystalline field into an $x$ and $y$ polarized component. The 388 bands can be ascribed to the $1 \mathrm{~s} \sigma_{\mathrm{g}} \rightarrow 3 \mathrm{p} \pi_{\mathrm{u}}$ transitions. The structureless long tail observed on the high energy side of the $388 \mathrm{~nm}$ bands (fig. 3) is probably the envelope of all the transitions to the higher $\pi$-like levels.

The oscillator strength $f^{\alpha \beta}\left(r_{\mathrm{AB}}, k_{0}\right)$ of a transition between two states $x$ and $\beta$ of our system immersed in a dielectric medium has been extensively discussed by $\operatorname{Lax}^{9}$ ) and later also by Herman et al. ${ }^{6}$ ). They showed that this value is strictly identical to the oscillator strength of the transition between the same states $\alpha$ and $\beta$ of the hydrogen molecular ion in the free space with an internuclear distance $R=r_{\mathrm{AB}} / k_{0}$. This justifies therefore the use of the values tabulated by Bates and collaborators ${ }^{10}$ ). The theoretical and measured values of the absolute oscillator strength for the $\mathrm{F}_{2}^{+}$center (for a detailed explanation see ${ }^{3}$ )) are summarized for most transitions in fig. 5 also. In spite of some inaccuracy due to experimental evaluation (especially when two or more bands are superimposed and cannot be resolved) the agreement is found surprisingly good over three orders of magnitude.

\section{References}

1) I. Schneider and H. Rabin, Phys. Rev. 140 (1965) Al983.

2) Y. Farge, G. Toulouse and M. Lambert, Compt. Rend. Acad. Sci. (Paris) 262B (1966) 1012;

J. Nahum and D. A. Wiegand, Phys. Rev. 154 (1967) 817:

J. Nahum, Phys. Rev, 158 (1967) 813.

K. Konrad and T. J. Neubert, J. Chem. Phys. 47 (1967) 4946:

J. Nahum, Phys. Rev. 174 (1968) 1000.

3) M. Aegerter and F. Lüty, to be published.

4) C. P. Pjerce, unpublished result quoted by D. B. Fitchen in Chapter 5 of Physics of Color Centers, Ed. W. B. Fowler (Academic Press Inc., New York, 1968).

5) W. B. Fowler and D. L. Dexter, Phys. Rev. 128 (1962) 2154.

6) R. Herman, M. C. Wallis and R. F. Wallis, Phys. Rev. 103 (1956) 87.

7) D. R. Bates, K. Ledsham and A. L. Stewart, Phil. Trans. Roy. Soc. A246 (1953) 215.

8) C. Gilbert, Phil. Mag. 16 (1933) 929.

9) M. Lax, 1954 Photoconductivity Conference (John Wiley, New York, 1956).

10) D. R. Bates, J. Chem. Phys. 19 (1951) 1122;

D. R. Bates, R. T. S. Darling, S. C. Hawe and A. L. Stewart, Proc. Phys. Soc. (London) A66 (1953) 1124, and A67 (1954) 533. 